La Revue

des Droits

de l'Homme

\section{La Revue des droits de l'homme}

Revue du Centre de recherches et d'études sur les droits fondamentaux

Actualités Droits-Libertés | 2018

\title{
Fin de vie : ne rien changer quand rien ne va
}

\section{Camille Bourdaire-Mignot et Tatiana Gründler}

\section{QpenEdition}

\section{Journals}

Édition électronique

URL : http://journals.openedition.org/revdh/4581

DOI : $10.4000 /$ revdh.4581

ISSN : 2264-119X

Éditeur

Centre de recherches et d'études sur les droits fondamentaux

\section{Référence électronique}

Camille Bourdaire-Mignot et Tatiana Gründler, « Fin de vie : ne rien changer quand rien ne va », La

Revue des droits de l'homme [En ligne], Actualités Droits-Libertés, mis en ligne le 04 août 2018, consulté le 23 avril 2019. URL : http://journals.openedition.org/revdh/4581 ; DOI : 10.4000/revdh.4581

Ce document a été généré automatiquement le 23 avril 2019.

Tous droits réservés 


\title{
Fin de vie : ne rien changer quand rien ne va
}

\author{
Camille Bourdaire-Mignot et Tatiana Gründler
}

1 Un sujet qui intéresse. La fin de vie est le deuxième sujet en termes de participation en ligne : il a attiré $22 \%$ des internautes et généré près de $24 \%$ des contributions réunies sur le site. Preuve de l'intérêt qu'il suscite, le Comité citoyen - mis en place pour porter un regard critique sur le déroulement des États généraux de la bioéthique - a décidé d'en faire l'un de ses thèmes de réflexion ${ }^{1}$. Enfin, 52 auditions ont concerné cette thématique. $\mathrm{Si}$, majoritairement, ce sont des associations - dont certaines très engagées sur le sujet (Alliance Vita, l'Union nationale des associations de parents de personnes handicapées mentales et leurs amis ou encore, dans un sens opposé, l'Association du droit de mourir dans la dignité) - qui ont été entendues, des représentants de l'ensemble des religions ainsi qu'un certain nombre d'institutions plus ou moins attendues sur ce thème ${ }^{2}$ et trois "sociétés savantes "- en réalité des professionnels de santé confrontés à des situations de fin de vie ${ }^{3}$ - apportèrent également leur éclairage sur ce sujet sensible.

2 Le Comité consultatif national d'éthique (CCNE) affirme dans son rapport de synthèse qu'"aucun consensus sociétal n'existe sur les questions du suicide assisté et de l'euthanasie, ni sur le site web, ni au cours des rencontres en régions, ni au cours des auditions des associations ». Cette conclusion paraît toutefois un peu hâtive. Certes, il existe des oppositions fondamentales sur l'aide à mourir mais d'autres analyses sur la fin de vie sont assez largement partagées.

3 Des divergences de vues. Le CCNE met en exergue des points de dissensions forts et difficilement conciliables entre ceux qui défendent le maintien de l'interdit absolu de tuer - et refusent donc toute évolution législative concernant une aide à mourir quelles qu'en soient les modalités - et ceux qui appellent à une prise en compte accrue de la liberté de disposer de son corps et de choisir sa mort - et demandent donc une reconnaissance juridique d'une aide à mourir. Le CCNE précise au demeurant, après avoir entendu seulement trois sociétés savantes, que les professionnels de santé expriment « une large et ferme opposition à ces pratiques ». À le suivre, l'affaire serait pliée. C'est sans compter le fait qu'un certain nombre de constats partagés sont occultés. 
Le constat partagé du mal mourir. Les Français ont confirmé lors de ces États généraux ce que différents rapports ont montré encore récemment, à savoir que l'on meurt mal en France $^{4}$. Cette situation est imputée en premier lieu à l'insuffisance de l'offre de soins palliatifs pour lesquels des moyens financiers d'envergure sont demandés 5 . D'autres facteurs sont mentionnés en second lieu dont le manque d'aide portée aux accompagnants des personnes en fin de vie, l'image négative de la vieillesse et le déni de la mort dans la société occidentale moderne.

5 La critique de la sédation profonde et continue jusqu'au décès. La sédation profonde et continue jusqu'au décès, principale innovation de la loi Claeys Leonetti de 2016, apparaît aux yeux de nombre de participants à ces États généraux comme un mauvais compromis. Allant trop loin pour ceux qui y voient une euthanasie déguisée, elle apparaît au contraire insuffisante à d'autres dès lors que l'appréciation de la souffrance et donc de la possibilité de la sédation relève du corps médical, ne laissant que peu de place à la volonté de la personne en fin de vie.

Une admission croissante de la reconnaissance d'une aide active à mourir. Sur le point le plus sensible que constitue l'aide active à mourir, force est de constater que le dissensus apparu lors de ces débats contraste avec les expressions populaires - certes sondagières - qui se sont montrées majoritairement favorables à la reconnaissance d'une aide active à mourir ${ }^{6}$.

7 Perspectives. La fin de vie ne semble pas promise à entrer dans le dispositif de révision périodique des lois bioéthiques. Il est vrai que le sujet n'est pas directement lié aux progrès scientifiques. Cependant, ce sont bien les avancées médicales qui déterminent les situations de fin de vie qui ne cessent d'interroger la société. Le sujet n'aurait donc servi qu'à donner corps aux débats citoyens? Il ne faudrait pas que cette question, dont le CCNE a pu déplorer qu'elle ait trop retenu l'attention des Français, n'ait finalement été qu'un écran de fumée. La vigueur des débats révèle que tant l'avis du CCNE que celui du législateur sont désormais attendus sur cette question. Peut-être la fin de vie trouvera-telle sa place dans une autre loi $^{7}$, une loi prudente, de compromis, à l'instar de la loi Veil de 1975 sur l'interruption volontaire de grossesse qui entendait répondre à une demande pourtant non majoritaire dans la population, dans la classe politique et chez les professionnels de santé.

CCNE, Rapport de synthèse du Comité consultatif national d'éthique - Opinions du Comité citoyen, juin 2018, $196 \mathrm{p}$.

Les Lettres « Actualités Droits-Libertés» (ADL) du CREDOF (pour s'y abonner) sont accessibles sur le site de la Revue des Droits de l'Homme (RevDH) - Contact 


\section{NOTES}

1. L'autre thème auquel il a réfléchi est la génomique en pré-conceptionnel.

2. Parmi les institutions impliquées sur cette question, on peut citer l'Académie de médecine ou le Conseil économique social et environnemental. Parmi les institutions moins attendues sur le sujet figurent le Conseil supérieur du notariat, le Conseil national des barreaux ou le Défenseur des droits qui s'est opposé, précise le CCNE, comme les familles religieuses, à la légalisation de l'assistance au suicide et/ou à l'euthanasie.

3. La Société française d'accompagnement des soins palliatifs, la Société française de néonatologie et la Société française d'anesthésie réanimation.

4. CESE, Fin de vie : la France à l'heure des choix, 10 avril 2018, http://www.lecese.fr/sites/default/ files/pdf/Avis/2018/2018_10_fin_vie.pdf ; CCNE, Avis n 128, Enjeux éthiques du vieillissement. Quel sens à la concentration des personnes âgées entre elles, dans des établissements dits d'hébergement? Quels leviers pour une société inclusive pour les personnes âgées? http://www.ccne-ethique.fr/sites/ default/files/publications/ccne_avis_128.pdf

5. Le Comité citoyen s'est dit alarmé par «le déficit des services de soins palliatifs en France » quand « trois patients sur quatre susceptibles d'en bénéficier n'y ont pas accès ".

6. «Bioéthique, ce qu'en disent les Français », La Croix, 3 janvier 2018.

7. Proposition de loi du député Jean-Louis Touraine, portant sur la fin de vie dans la dignité, $\mathrm{n}$ - 185 du 27 septembre 2017. V. la tribune publiée dans Le Monde, 28 février 2018, « Fin de vie : 156 députés font pression pour une nouvelle loi ».

\section{RÉSUMÉS}

Depuis 2005 et la première loi consacrée à la fin de vie, le législateur français a, de façon constante, souhaité encourager le recours aux soins palliatifs afin d'améliorer les conditions de la fin de vie et faire ainsi disparaitre les demandes d'abréger la vie. Visiblement, la voie choisie n'a pas permis de répondre à toutes les situations individuelles. Ainsi de nombreux Français se rendent à l'étranger, en Suisse ou en Belgique, pour tirer avantage d'une législation plus libérale c'est-à-dire concrètement d'un suicide assisté ou d'une euthanasie. Bien que la question de la fin de vie ne soit pas fondamentalement renouvelée par des progrès scientifiques ou médicaux, il est apparu qu'elle ne pouvait être éludée et fut donc inscrite à l'ordre du jour des États généraux de la bioéthique.

\section{AUTEURS}

\section{CAMILLE BOURDAIRE-MIGNOT}

Maître de conférences en droit privé, CEDCACE, Université Paris Nanterre 


\section{TATIANA GRÜNDLER}

Maître de conférences en droit public, CTAD-CREDOF, Université Paris Nanterre 